\title{
DIRICHLET INTEGRAL AND STAR-FUNCTION INEQUALITIES
}

\author{
J. R. QUINE
}

\begin{abstract}
Let $\lambda(z)$ be a smooth function in an annulus, $\tilde{\lambda}(z)$ its symmetric rearrangement and $\lambda^{*}(z)$ its star-function. A formula is proved relating $\Delta \lambda^{*}, \Delta \lambda$, and the Dirichlet integrands of $\lambda$ and $\tilde{\lambda}$. The formula shows the relationship between Dirichlet integral inequalities and the subharmonicity of $\lambda^{*}$ for subharmonic $\lambda$, and gives an explicit formula for $\Delta \lambda^{*}$.
\end{abstract}

The purpose of this paper is to continue the study initiated in [6 and 7] of the relationship between the Dirichlet integral inequalities of Pólya and Szegö [5] and the star-function techniques of Baernstein $[1,2]$. There we considered a function $f$ analytic in an annulus and studied the function $\partial(\log |f|)^{*} / \partial \log z$ showing that it could be viewed as a symmetrization of the function $f$. Let $\Delta=\partial^{2} / \partial l^{2}+\partial^{2} / \partial \theta^{2}$ where $l=\log |z|$ and $\theta=\arg z$. We showed that $\Delta(\log |f|)^{*}$ exists in the usual sense almost everywhere and we gave an explicit expression for it in terms of the partial derivatives of $\log |f|$. As a consequence, the inequality $\Delta(\log |f|)^{*} \geqslant 0$ was seen to be equivalent to a nonintegrated version of the Dirichlet integral inequality between $\log |f|$ and its symmetrization. The techniques made heavy use of the harmonicity of $\log |f|$ and of the conjugate function $\arg f$.

In this paper we extend our results to include any smooth function $\lambda(z)$ satisfying fairly general regularity conditions. We give a formula for $\Delta \lambda^{*}$ in terms of the Dirichlet integrand of $\lambda$ and its rearrangement $\tilde{\lambda}$ and an integral involving $\Delta \lambda$ (Theorem 2). The proof is by explicit computation of all terms involved. When applied to subharmonic $\lambda$ this formula gives the subharmonicity of $\lambda^{*}$ which is seen to be a consequence of the inequality $\Delta \lambda \geqslant 0$ and the unintegrated Dirichlet integral inequality. The unintegrated Dirichlet inequality is stated in Theorem 1.

Let $\lambda(z)$ be a smooth function defined in an annulus $r_{1}<|z|<r_{2}$. It will be convenient to think of $\lambda=\lambda(l, \theta)$ as a function with period $2 \pi$ in the variable $\theta$. The graph $W$ of $\lambda$ will be thought of as lying in a $(l, \theta, \lambda)$ plane with points $(l, \theta+2 \pi k ; \lambda)$ identified for integers $k$.

For fixed $l_{0}, \lambda_{0}$ let $E\left(l_{0}, \lambda_{0}\right)$ be the set of points $\left(l_{0}, \theta, \lambda_{0}\right)$ on $W$ with $\lambda \geqslant \lambda_{0}$. Let $E_{\theta}\left(l_{0}, \lambda_{0}\right)$ be the set of $\theta$ in the interval $[-\pi, \pi]$ such that $\left(l_{0}, \theta, \lambda_{0}\right)$ is in $E\left(l_{0}, \lambda_{0}\right)$. Let $W_{+}^{*}$ be the set of points of the form $\left(l, \frac{1}{2}\left|E_{\theta}(l, \lambda)\right|, \lambda\right), E_{\theta}(l, \lambda) \neq \varnothing$. Let $W_{-}^{*}$ be the set of points of the form $(l, \theta, \lambda)$ with $(l,-\theta, \lambda)$ in $W_{+}^{*}$. Then $W^{*}=W_{+}^{*} \cup W_{-}^{*}$ determines the graph of the rearranged function $\tilde{\lambda}$ (see [2]).

We will consider pairs $\left(l_{0}, \lambda_{0}\right)$ such that $W$ intersects the line $l=l_{0}, \lambda=\lambda_{0}$ transversely. This means that $\lambda\left(l_{0}, \theta\right)=\lambda_{0}$ at a finite number of values of $\theta$ at each

Received by the editors June 15, 1984 and, in revised form, February 12, 1985

1980 Mathematics Subject Classification. Primary 31A05; Secondary 30D35, 30C55.

(C)1986 American Mathematical Society $0002-9939 / 86 \$ 1.00+\$ .25$ per page 
of which $\lambda_{\theta}\left(l_{0}, \theta\right) \neq 0$. We will refer to such an $\left(l_{0}, \lambda_{0}\right)$ as regular. For regular $\left(l_{0}, \lambda_{0}\right)$, let $W\left(l_{0}, \lambda_{0}\right)$ be the set of points $\left(l_{0}, \theta, \lambda_{0}\right)$ on $W$. Near $\left(l_{0}, \lambda_{0}\right)$ write $W(l, \lambda)=\left\{\left(l, \theta_{k}, \lambda\right) \mid k=1,2, \ldots, 2 n\right\}$ where $\theta_{k}=\theta_{k}(l, \lambda)$ is chosen to vary smoothly with $(l, \lambda)$. Define $\varepsilon_{k}=\varepsilon\left(l, \theta_{k}, \lambda\right)=-\operatorname{sgn} \lambda_{\theta}\left(l, \theta_{k}\right)$, then the 0 -chain $\sum_{p \in W(l, \lambda)} \varepsilon_{k} p$ may be thought of as the boundary of the 1-chain $E(l, \lambda)$. For suitably chosen $\theta_{k}$, if we write

$$
\theta(l, \lambda)=\frac{1}{2} \sum_{k=1}^{2 n} \varepsilon_{k} \theta_{k}(l, \lambda),
$$

then this equation can be thought of as a local parameterization of the surface $W_{+}^{*}$. Since $\theta$ is a decreasing function of $\lambda$ for fixed $l,(l, \theta)$ also gives local coordinates on $W_{+}^{*}$. Note that for regular $(l, \lambda), W^{*}(l, \lambda)$ is the union of singletons $W_{+}^{*}(l, \lambda)$ and $W_{-}^{*}(l, \lambda)$.

Define

$$
\lambda^{*}(l, \lambda)=\int_{E(l, \lambda)} \lambda(l, t) d t
$$

The star-function $\lambda^{*}$ may be thought of as a function on $W_{+}^{*}$. Transferring to $(l, \theta)$ coordinates it may be thought of as a function in the upper-half plane near $\exp \left(l_{0}+i \theta\left(l_{0}, \lambda_{0}\right)\right)$ where $\left(l_{0}, \lambda_{0}\right)$ is regular. (The global situation will be discussed later.)

On $W$ or $W^{*}$ we will frequently need to alternate between the variables $(l, \theta)$ and $(l, \lambda)$ at points where $d l \wedge d \lambda$ is nonzero. This is most conveniently done by using the 2-forms $d \theta \wedge d \lambda, d l \wedge d \lambda$, and $d l \wedge d \theta$ on the surface. For example, $d f \wedge d l / d \lambda \wedge d l$ can be interpreted as the partial derivative of $f$ with respect to $\lambda$ leaving $l$ fixed on the surface, while $d f \wedge d \theta / d \lambda \wedge d \theta$ is the partial derivative with respect to $\lambda$ leaving $\theta$ fixed.

Let $D(\lambda)=\left(\lambda_{l}^{2}+\lambda_{\theta}^{2}\right) d l \wedge d \theta$ denote the Dirichlet integrand. The basic Dirichlet integral inequality of Pólya and Szegö may be stated in its unintegrated version as follows.

TheOrem 1 (Pólya-Szego). For regular $(l, \lambda)$,

$$
\sum_{W(l, \lambda)} \frac{D(\lambda)}{|d \lambda \wedge d l|} \geqslant \sum_{W^{*}(l, \lambda)} \frac{D(\lambda)}{|d \lambda \wedge d l|} .
$$

The proof given in Pólya-Szegö [5, Note A] is derived from the inequality

$$
\sum_{W(l, \lambda)} \frac{d S}{|d \lambda \wedge d l|} \geqslant \sum_{W^{*}(l, \lambda)} \frac{d S}{|d \lambda \wedge d l|}
$$

where $d S=\left((d \lambda \wedge d l)^{2}+(d l \wedge d \theta)^{2}+(d \lambda \wedge d \theta)^{2}\right)^{1 / 2}$ is the element of surface area. Inequality (4) is due to Steiner and follows from the triangle inequality. By (1), we have

$$
\sum_{W(l, \lambda)}\left|\frac{d \theta \wedge d l}{d \lambda \wedge d l}\right|=\sum_{W^{*}(l, \lambda)}\left|\frac{d \theta \wedge d l}{d \lambda \wedge d l}\right|
$$


and

$$
\sum_{W(l, \lambda)} \varepsilon \frac{d \theta \wedge d \lambda}{d l \wedge d \lambda}=\sum_{W^{*}(l, \lambda)} \varepsilon \frac{d \theta \wedge d \lambda}{d l \wedge d \lambda}
$$

Theorem 1 may be obtained by subtracting (5) from (4), replacing $\lambda$ by $\delta \lambda$, dividing by $\delta^{2} / 2$ and letting $\delta \rightarrow 0$.

Another approach to (3) is by direct computation as in Hayman [4, p. 77]. We will compute an expression for

$$
F(l, \lambda)=\sum_{W(l, \lambda)} \frac{D(\lambda)}{|d \lambda \wedge d l|}-\sum_{W^{*}(l, \lambda)} \frac{D(\lambda)}{|d \lambda \wedge d l|},
$$

to prove Theorem 2 . We have

$$
\begin{aligned}
\frac{D(\lambda)}{|d \lambda \wedge d l|} & =\left(\left|\frac{d \lambda \wedge d \theta}{d l \wedge d \theta}\right|^{2}+\left|\frac{d \lambda \wedge d l}{d \theta \wedge d l}\right|^{2}\right)\left|\frac{d l \wedge d \theta}{d \lambda \wedge d l}\right| \\
& =\left|\frac{d \theta \wedge d \lambda}{d l \wedge d \lambda}\right|^{2}\left|\frac{d \theta \wedge d l}{d \lambda \wedge d l}\right|^{-1}+\left|\frac{d \theta \wedge d l}{d \lambda \wedge d l}\right|^{-1}
\end{aligned}
$$

Let $P$ be the point $W_{+}^{*}(l, \lambda)$. Then using (5), (6), (7) and the symmetry $W^{*}$ we have

$$
\begin{aligned}
\sum_{W^{*}(l, \lambda)} \frac{D(\lambda)}{|d \lambda \wedge d l|} & =\left.\left(2\left|\frac{d \theta \wedge d \lambda}{d l \wedge d \lambda}\right|^{2}\left|\frac{d \theta \wedge d l}{d \lambda \wedge d l}\right|^{-1}+2\left|\frac{d \theta \wedge d l}{d \lambda \wedge d l}\right|^{-1}\right)\right|_{P} \\
& =\left[2\left(\frac{1}{2} \sum_{W(l, \lambda)} \varepsilon \frac{d \theta \wedge d \lambda}{d l \wedge d \lambda}\right)^{2}\left(\frac{1}{2} \sum_{W(l, \lambda)}\left|\frac{d \theta \wedge d l}{d \lambda \wedge d l}\right|\right)^{-1}\right. \\
& \left.+2\left(\frac{1}{2} \sum_{W(l, \lambda)}\left|\frac{d \theta \wedge d l}{d \lambda \wedge d l}\right|\right)^{-1}\right]
\end{aligned}
$$

Thus

(8) $F(l, \lambda)=\left[\sum \theta_{l}^{2}\left|\theta_{\lambda}\right|^{-1}-\left(\sum \varepsilon \theta_{l}\right)^{2}\left(\sum\left|\theta_{\lambda}\right|\right)^{-1}\right]+\left[\sum\left|\theta_{\lambda}\right|^{-1}-4\left(\sum\left|\theta_{\lambda}\right|\right)^{-1}\right]$

where $\theta_{l}=d \theta \wedge d \lambda / d l \wedge d \lambda, \theta_{\lambda}=d \theta \wedge d l / d \lambda \wedge d l$ and the sum is over $W(l, \lambda)$. Now each term in brackets in (8) is seen to be nonnegative by the Cauchy-Schwarz inequality, again demonstrating Theorem 1 .

We now prove

THEOREM 2. If $(l, \lambda)$ is regular with $\lambda=\tilde{\lambda}(l, \theta)$, then

$$
\begin{aligned}
\Delta \lambda^{*}(l, \theta)= & \int_{E(l, \lambda)} \Delta \lambda(l, t) d t+\sum_{W(l, \lambda)} \frac{D(\lambda)}{|d \lambda \wedge d l|} \\
& -\sum_{W^{*}(l, \lambda)} \frac{D(\lambda)}{|d \lambda \wedge d l|}
\end{aligned}
$$

Proof. The star-function may be written

$$
\lambda^{*}(l, \theta)=\int_{-\theta}^{\theta} \tilde{\lambda}(l, t) d t
$$


and so differentiating twice, we easily get

$$
\lambda_{\theta \theta}^{*}=2 \tilde{\lambda}_{\theta}=\left.2 \frac{d \lambda \wedge d l}{d \theta \wedge d l}\right|_{P} .
$$

Now differentiating (2) we get (for $\lambda^{*}$ as a function of $(l, \theta)$ )

$$
\lambda_{l}^{*}=\int_{E(l, \lambda)} \lambda, d \theta+\lambda \sum_{k} \varepsilon_{k} \frac{d \theta_{k} \wedge d \theta}{d l \wedge d \theta}
$$

where in the second term we think of the $\theta_{k}$ as functions on $W^{*}$. Since $\sum \varepsilon_{k} \theta_{k}=2 \theta$, the second term in (11) is identically zero. Differentiating once more we get

$$
\begin{aligned}
\lambda_{l l}^{*} & =\int_{E(l, \lambda)} \lambda_{l l} d \theta+\sum_{k} \varepsilon_{k} \lambda \frac{d \theta_{k} \wedge d \theta}{d l \wedge d \theta} \\
& =\int_{E(l, \lambda)} \lambda_{l l} d \theta+\sum_{k} \varepsilon_{k} \frac{d \lambda \wedge d \theta_{k}}{d l \wedge d \theta_{k}} \frac{d \theta_{k} \wedge d \theta}{d l \wedge d \theta} \\
& =\int_{E(l, \lambda)} \lambda_{l l} d \theta+A
\end{aligned}
$$

where $A$ denotes the sum above.

To derive an expression for $A$, we need an expression for $d \theta_{k} \wedge d \theta / d l \wedge d \theta$. First write

$$
d \lambda=\frac{d \lambda \wedge d \theta_{k}}{d l \wedge d \theta_{k}} d l+\frac{d \lambda \wedge d l}{d \theta_{k} \wedge d l} d \theta_{k} .
$$

Taking the wedge product with $d \theta$ and dividing by $d l \wedge d \theta$,

$$
\frac{d \lambda \wedge d \theta}{d l \wedge d \theta}=\frac{d \lambda \wedge d \theta_{k}}{d l \wedge d \theta_{k}}+\frac{d \lambda \wedge d l}{d \theta_{k} \wedge d l} \frac{d \theta_{k} \wedge d \theta}{d l \wedge d \theta}
$$

Solving, get

$$
\frac{d \theta_{k} \wedge d \theta}{d l \wedge d \theta}=\left(\frac{d \lambda \wedge d l}{d \theta_{k} \wedge d l}\right)^{-1}\left(\frac{d \lambda \wedge d \theta}{d l \wedge d \theta}-\frac{d \lambda \wedge d \theta_{k}}{d l \wedge d \theta_{k}}\right) .
$$

Substituting in the expression above for $A$, get

$$
\begin{aligned}
A & =\sum_{k} \varepsilon_{k} \frac{d \lambda \wedge d \theta_{k}}{d l \wedge d \theta_{k}}\left(\frac{d \lambda \wedge d l}{d \theta_{k} \wedge d l}\right)^{-1}\left(\left.\frac{d \lambda \wedge d \theta}{d l \wedge d \theta}\right|_{P}-\frac{d \lambda \wedge d \theta_{k}}{d l \wedge d \theta_{k}}\right) \\
& =\left.\left(\frac{d \lambda \wedge d \theta}{d l \wedge d \lambda} \frac{d \lambda \wedge d l}{d l \wedge d \theta}\right)\right|_{P} \sum_{k} \frac{d \theta_{k} \wedge d \lambda}{d l \wedge d \lambda}+\sum_{k}\left|\frac{d \theta_{k} \wedge d \lambda}{d l \wedge d \lambda}\right|^{2}\left|\frac{d \lambda \wedge d l}{d \theta_{k} \wedge d l}\right| .
\end{aligned}
$$

Using (5), (6) and symmetry as in the computation of (8), we see that $A$ is identical to the first term in brackets in (8).

By (10) and (12), we get

$$
\begin{aligned}
\Delta \lambda^{*} & =\int_{E(l, \lambda)} \lambda_{l l} d \theta+\left.2 \frac{d \lambda \wedge d l}{d \theta \wedge d l}\right|_{P}+A \\
& =\int_{E(l, \lambda)} \Delta \lambda d \theta-\int_{E(l, \lambda)} \lambda_{\theta \theta} d \theta-2\left|\frac{d \theta \wedge d l}{d \lambda \wedge d l}\right|_{P}^{-1}+A \\
& =\int_{E(l, \lambda)} \Delta \lambda d \theta+\left(\sum_{W(l, \lambda)}\left|\frac{d \lambda \wedge d l}{d \theta \wedge d l}\right|-2\left|\frac{d \theta \wedge d l}{d \lambda \wedge d l}\right|_{P}^{-1}\right)+A
\end{aligned}
$$


The expression in parentheses is seen using (5) to be identical to the second term in brackets in (8). This completes the proof of the theorem.

We remark that an expression for $F$ can be obtained in the $(l, \theta)$ coordinate system. First write

$$
\frac{d \theta \wedge d \lambda}{d l \wedge d \lambda}=\frac{d \theta \wedge d \lambda}{d \theta \wedge d l} \frac{d \theta \wedge d l}{d l \wedge d \lambda}=-\frac{d \lambda \wedge d \theta}{d l \wedge d \theta}\left(\frac{d \lambda \wedge d l}{d \theta \wedge d l}\right)^{-1}
$$

and

$$
\frac{d \theta \wedge d l}{d \lambda \wedge d l}=\left(\frac{d \lambda \wedge d l}{d \theta \wedge d l}\right)^{-1}
$$

Substituting the above in (8), get

$$
\begin{aligned}
F(l, \lambda)= & \sum \lambda_{l}^{2}\left|\lambda_{\theta}\right|^{-1}-\left(\sum \lambda_{l}\left|\lambda_{\theta}\right|^{-1}\right)^{2}\left(\sum\left|\lambda_{\theta}\right|^{-1}\right)^{-1} \\
& +\sum\left|\lambda_{\theta}\right|-4\left(\sum\left|\lambda_{\theta}\right|^{-1}\right)^{-1}
\end{aligned}
$$

where

$$
\lambda_{\theta}=\frac{d \lambda \wedge d l}{d \theta \wedge d l}, \quad \lambda_{l}=\frac{d \lambda \wedge d \theta}{d l \wedge d \theta}
$$

Theorem 2 shows the general principle behind the local subharmonicity of the star function. If $\Delta \lambda \geqslant 0$ everywhere, then clearly $\Delta \lambda^{*} \geqslant 0$ at points corresponding to regular $(l, \lambda)$. There remains the question of how this applies to the subharmonicity of $\lambda^{*}$ at points where $\Delta \lambda^{*}$ is undefined, i.e., whether the subharmonicity of $\lambda^{*}$ for subharmonic $\lambda$ can be proven using the computation of the Laplacian in Theorem 2 .

We will show that subharmonicity of $\lambda^{*}$ for subharmonic $\lambda$ can be proved using the computation of the Laplacian in Theorem 2. Recall that if $\phi$ is a locally integrable function then there is associated to it a distribution $T_{\phi}$ (see Donoghue [3, p. 92]). We now state

THEOREM 3. If 0 is a regular value of $\lambda_{\theta}$, then the distribution corresponding to the function $\Delta \lambda^{*}$ in Theorem 2 is the distributional Laplacian of $\lambda^{*}$.

Proof. By hypothesis, the set $S$ of points $(l, \theta)$ such that $\lambda_{\theta}(l, \theta)=0$ is a smooth 1 -submanifold of the $(l, \theta)$ plane. Let $S^{\prime}$ be the set of points $(l, \theta)$ with $0<\theta<\pi$ such that $\tilde{\lambda}(l, \theta)=\lambda(l, t)$ for some $(l, t) \in S$, then $S^{\prime}$ consists of the union of a finite number of smooth curves. Now if $D$ is the strip $0<\theta<\pi$ then $\Delta \lambda^{*}$ is defined by Theorem 2 in $D-S^{\prime}$. By (10) and (11) we see that the first partials of $\lambda^{*}$ are continuous in $D$, hence $\lambda^{*} \in C^{1}(D) \cap C^{2}\left(D-S^{\prime}\right)$. Under these conditions it is easy to see using Green's theorem that $T_{\Delta \lambda^{*}}=\Delta T_{\lambda^{*}}$, where the second $\Delta$ indicates the distributional Laplacian. (Green's theorem shows that the equation holds on each subregion of $D-S^{\prime}$ except for a term involving integration over the boundary. These terms cancel because $\lambda^{*} \in C^{1}(D)$.) This completes the proof of the theorem. We now use Theorem 3 to prove

TheOREM 4 (BAERnSTEIN). If $\lambda$ is subharmonic then $\lambda^{*}$ is subharmonic. 
Proof. We will show that $\Delta T_{\lambda^{*}} \geqslant 0$ which implies the subharmonicity of $\lambda^{*}$ (Donoghue [3, p. 128]). Since it can be easily shown that

$$
\left\|\lambda_{1}^{*}-\lambda_{2}^{*}\right\|_{\infty} \leqslant 2 \pi\left\|\lambda_{1}-\lambda_{2}\right\|_{\infty}
$$

and since the smooth subharmonic functions are dense in the subharmonic functions, we may assume without loss of generality that $\lambda$ is smooth. By Sard's Theorem the regular values of $\lambda_{\theta}$ are dense, so the $\varepsilon$ may be chosen arbitrarily small so that 0 is a regular value of $\lambda_{\theta}+\varepsilon=(\lambda+\varepsilon \theta)_{\theta}$. The function $\lambda+\varepsilon \theta$ is also subharmonic, so by Theorem $3, \Delta T_{(\lambda+\varepsilon \theta)^{*}} \geqslant 0$. Letting $\varepsilon \rightarrow 0$, get $\Delta T_{\lambda^{*}} \geqslant 0$. This completes the proof.

In conclusion we remark that in Pólya-Szegö [5, Note A] it was pointed out that the principle of diminution of surface area and of Dirichlet integral holds not only globally, but also locally for all the surface elements $W(l, \lambda)$. The power of star-function techniques appears to be a consequence of the fact that it is equivalent to the stronger, unintegrated form of the Dirichlet integral inequality.

\section{REFERENCES}

1. A. Baernstein, Proof of Edrei's spread conjecture, Proc. London Math. Soc. (3) 26 (1973), 418-434.

2. 139-169.

3. W. Donoghue, Distributions and Fourier transforms, Academic Press, New York, 1969.

4. W. K. Hayman, Multivalent functions, Cambridge Univ. Press, 1967.

5. G. Pólya and G. Szegö, Isoperimetric inequalities in mathematical physics, Princeton Univ. Press, Princeton, N.J., 1951.

6. J. R. Quine, Circular symmetrization and the * function, Complex Variables Theory and Applications 2 (1984), 341-352.

7. The Laplacian of the * function, Contemporary Mathematics, Topics in Complex Analysis, vol. 38, Amer. Math. Soc., Providence, R.I., 1985, pp. 121-125.

Department of Mathematics, Florida State University, Tallahassee, Florida 32306

Institute for Advanced Study, School of Mathematics, Princeton, New Jersey 08540 